\title{
Mapping diplosporous apomixis in tetraploid Tripsacum: one gene or several genes?
}

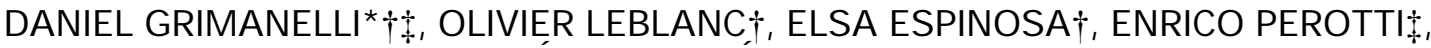 \\ DIEGO GONZALEZ DE LEON $\ddagger$ \& YVES SAVIDAN $\dagger$ \\ †ORSTOM, Institut Français de Recherche Scientifique pour le Développement en Coopération and \$CIMMYT, \\ International Maize and Wheat Improvement Center, CIMMYT Applied Biotechnology Center, Apda 6-641, 06600 \\ Mexico DF, Mexico
}

\begin{abstract}
Polyploids in Tripsacum, a wild relative of maize, reproduce through the diplosporous type of apomixis, an asexual mode of reproduction through seeds. Diplosporous apomixis involves both the failure of meiosis and the parthenogenetic development of the unreduced gametes, resulting in progenies that are exact genetic copies of the mother plant. Apomixis is believed to be controlled by one single dominant allele, responsible for the whole developmental process. Construction of a linkage map for the chromosome controlling diplosporous apomixis in Tripsacum was carried out in both tetraploid-apomictic and diploid-sexual Tripsacum species using maize restriction fragment length polymorphism (RFLP) probes. A high level of collinearity was observed between the Tripsacum chromosome carrying the control of apomixis and a duplicated segment in the maize genome. In the apomictic tetraploid, there was a strong restriction to recombination, as compared to the corresponding genomic segment in sexual plants and maize. This suggests that apomixis, although inherited as a single Mendelian allele, might really be controlled by a cluster of linked loci. The analysis also revealed the tetrasomic nature of the inheritance of the chromosomal segment controlling apomixis, which contradicts the usually accepted hypothesis of an allopolyploid origin of apomictic species. The implications of these data for the transfer of apomixis into cultivated crops are discussed, and a new approach to studying the genetics of apomixis, based on comparative mapping, is proposed.
\end{abstract}

Keywords: apomixis, comparative mapping, diplospory, Tripsacum, Zea mays.

\section{Introduction}

Gametophytic apomixis in higher plants refers to several mechanisms of asexual reproduction through seeds (Stebbins, 1950; Nogler, 1984b). In all cases, the apomictic process bypasses both meiosis and egg cell fertilization, producing offspring which are exact genetic replicas of the mother plant. Two major types of gametophytic apomixis have been described, namely diplosporous apomixis and aposporous apomixis, depending on the origin of the unreduced megagametophytes. In aposporous apomicts one or more unreduced female gametophytes form mitotically from somatic nucellar cells, whereas the legitimate sexual line generally aborts. Diplospory results from meiotic failure in megasporocytes that develop

*Correspondence: ORSTOM-CIMMYT, Apdo 6-641, 06600 Mexico DF, Mexico. E-mail: dgrimanelli@cimmyt.mx

(c) 1998 The Genetical Society of Great Britain. into mature, unreduced female gametophytes. Typically, apomixis is a facultative phenomenon in which an apomictic plant usually produces a few meiotic embryos beside a majority of ameiotic (apomeiotic, after Renner, 1916) embryos. Furthermore, apomicts are usually restricted to polyploids (Nogler, 1984b).

Apomixis is a genetically controlled character (Nogler, 1984b). Despite decades of research, this control remains poorly understood and only two propositions have received a broad acceptance. First, most authors agree upon a surprisingly simple monogenic inheritance of aposporous apomixis, based on segregation analyses conducted in species as diverse as Panicum maximum (Savidan, 1982), Ranunculus auricomus (Nogler, 1984a) or Brachiaria ssp. (do Valle \& Savidan, 1996), where the formation of unreduced embryo sacs segregates as a single dominant allele. Regarding the genetic control of 
diplospory, no conclusive studies have been conducted, apart from work in Taraxacum (review by Mogie, 1992) which shows that the gene or genes for diplospory are located on a single chromosome, and in Tripsacum (Leblanc et al., 1995a) which suggests Mendelian inheritance. Secondly, it is also widely accepted that apomictic species are of allopolyploid origin, formed after hybridization between two diploid relatives (Gustafsson, 1946-47; Asker \& Jerling, 1992).

In this paper, we report our current attempt to tag the genes responsible for the diplosporous apomictic mode of reproduction in Tripsacum, the closest wild apomictic relative of Zea. The results reported here are used to discuss current hypotheses on the genetic control of apomixis, and tend to question, at least in Tripsacum, both the one-dominant-gene hypothesis and the allopolyploid origin of apomictic plants.

\section{Materials and methods}

Plant material

Two segregating populations were used. An $F_{1}$ population segregating for diplospory was derived from hybridization between a maize (Zea mays L.) plant $(2 n=20)$ from CIMMYT population 34 as the pistillate parent, and CIMMYT Tripsacum dactyloides (L.) L. accession 65-1234, an apomictic tetraploid $(2 n=4 \mathrm{x}=72)$ as the male parent. All the hybrids recovered were $2 n=46$ combining 36 chromosomes from the Tripsacum parent and 10 from the maize parent $(10 \mathrm{Mz}+36 \mathrm{Td})$. This population, consisting of 51 plants, was found to be segregating $1: 1$ for modes of reproduction, diplospory vs. meiosis (Leblanc et al., 1995a). An interspecific mapping population at the diploid level consisting of 175 individual $F_{1}$ plants was derived from controlled hybridization between two sexual diploid $(2 n=36)$ accessions. Tripsacum maizar Hern. and Randolph, CIMMYT accession number 99-1114, was used as the pistillate parent, and Tripsacum dactyloides var. meridionale de Wet and Timothy, CIMMYT accession number 575-5136, as the male parent. Fifty-six plants were randomly selected for RFLP analysis.

\section{Determination of modes of reproduction}

Analysis of callose deposition during megasporogenesis (sucrose-aniline blue clearing procedure combined with epifluorescence microscopy), a reliable indicator of meiosis, was used for cytoembryo- logical determination of diplosporous reproduction, as described in Leblanc et al. (1995b).

\section{RFLP detection}

DNA was extracted using a CTAB procedure (Hoisington et al., 1994), digested using restriction endonucleases HindIII or EcoRI, electrophoresed and transferred onto uncharged nylon membranes. Extraction, digestion, hybridizations, stringency washes, chemiluminescent detection and exposure were performed according to Hoisington et al. (1994). Maize RFLP clones were kindly supplied by the University of Missouri, Columbia.

\section{Linkage analyses}

The identification of RFLP markers linked to diplospory in the maize by tetraploid Tripsacum $\mathrm{F}_{1}$ population has been reported elsewhere (Leblanc et al., 1995a). The conditions for the detection of markers linked to diplospory were as follows: polymorphism between the maize and Tripsacum parents, segregation as a single-dose RFLP heterozygous marker in the $F_{1} s$, and linkage with the apomictic mode of reproduction. Note that even though the mapping population was derived from an interspecific cross, the gametic generation used for linkage analysis corresponds only to the male gametes in the Tripsacum parent, so that the interspecific nature of the cross does not interfere with the estimation of linkage.

The same probes were mapped both on the $F_{1}$ population between maize and tetraploid Tripsacum, and on the diploid $\mathrm{F}_{1}$ population. Chi-squared tests were used to detect linkage, and estimations of recombination frequencies were obtained following Ritter et al. (1990).

Nature of the inheritance of the segment controlling diplospory

RFLP markers are codominant, i.e. they potentially allow the identification of all the alleles at one given locus. Therefore, RFLPs can be used to discriminate between allo- and autopolyploid segregation in the progeny of a tetraploid individual: the various alleles at a given locus should segregate, with two pairs of alleles being totally or partially in repulsion in the case of allotetraploidy (disomic inheritance), whereas random or near-random associations between the alleles are expected for autotetraploid segments (polysomic inheritance).

(c) The Genetical Society of Great Britain, Heredity, 80, 33-39. 


\section{Results}

\section{Mapping displospory in tetraploid Tripsacum}

Significant corrections to previously reported results of linkage between diplospory and molecular markers could be made through careful review of the data, both at the molecular genotype and reproductive phenotype levels. The current status of the mapping effort is shown in Fig. 1. Among the plants that had been analysed by Leblanc et al. (1995a), only those for which both the mode of reproduction and the RFLP genotypes could be confirmed were selected. Four plants that had been previously classified as apomicts and recombined for the closest RFLP alleles, could not be confirmed because they either had failed to flower again, or had died. Hence, the recombination estimate varies between complete linkage (estimation without including the doubtful individuals) and 8 per cent $( \pm 3.7)$.

\section{Mapping in diploid-sexual Tripsacum and comparative mapping to maize}

The loci that could be mapped in the maize-Tripsacum population segregating for modes of reproduction were also mapped in the diploid-sexual mapping population (Fig. 1). All the markers listed on the diploid linkage group could be mapped on a single parent, so that the results presented here correspond only to the male gametes. The same probes detected the same linkage groups at both ploidy levels in Tripsacum. As expected, this chromosomal segment showed a good level of collinearity with homoeologous maize segments, probably located on chromosome 6L (Fig. 1), or the corresponding duplication on 8L (Table 1). This segment is also duplicated on chromosome 3 in maize, but none of the probes used here has been mapped on chromosome 3 . Note that marker order is not entirely consistent between the Tripsacum segment and maize chromosome 6L, some inverted loci being detected. Interestingly, recombination patterns were remarkably different between the two Tripsacum ploidy levels. Although the RFLP loci were completely linked in the tetraploid-apomict, extensive recombination could be observed in the sexual-diploids and the published maize maps.

\section{Tetrasomic inheritance of the segment controlling diplospory}

Figure 2 shows the pattern of segregation in the maize-Tripsacum $F_{1}$ population of four alleles revealed by umc28, one of the probes detecting a locus linked with diplospory. Each of the four alleles segregates $\quad 1: 1 \quad\left(\chi_{1}^{2}=0.69, \quad P=0.41 ; \quad \chi_{1}^{2}=0.00\right.$, $P=1.00 ; \quad \chi_{1}^{2}=0.69, \quad P=0.41 ; \quad \chi_{1}^{2}=0.08, \quad P=0.78$, respectively), meaning that each represents a singledose allele. The more likely interpretation of this pattern is that the four alleles observed at the tetraploid level belong to a single locus. More complex interpretations could be considered, with the four alleles being distributed among two, three or four loci, but they would imply null RFLP alleles at these loci. Assuming, therefore, that the four alleles belong to one single locus, they can be used
Fig. 1 Linkage map of diplospory in apomictic tetraploid Tripsacum, and location of the corresponding loci in diploid sexual Tripsacum and maize chromosome $6 \mathrm{~L}$.

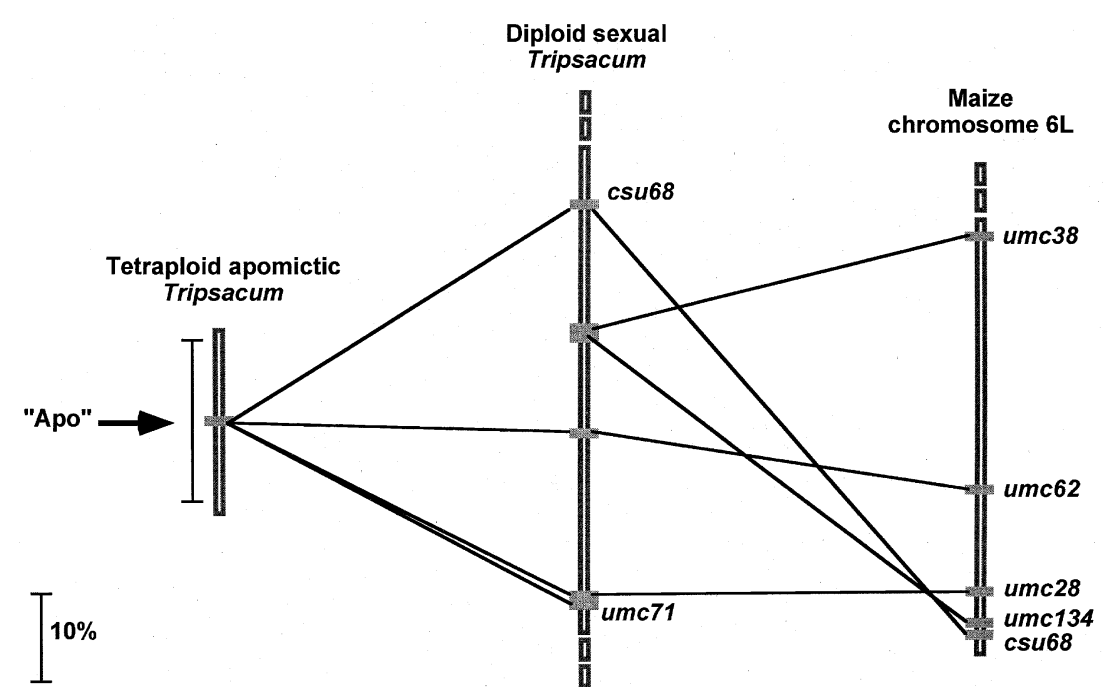


Table 1 Known duplications in the maize genome of the loci linked to diplospory in the tetraploid Tripsacum. (Source: Maize Genet. Coop. Newsletter, 1989-96, pers. comm.)

\begin{tabular}{ll}
\hline Clone & Location in maize \\
\hline umc38 & $6 \mathrm{~L}, 8 \mathrm{~L}$ \\
umc62 & $6 \mathrm{~L}$ \\
umc71 & $6 \mathrm{~L}, 8 \mathrm{~L}$ \\
umc28 & $6 \mathrm{~L}$ \\
csu68 & $6 \mathrm{~L}, 8 \mathrm{~L}$ \\
\hline
\end{tabular}

to determine disomic vs. tetrasomic inheritance. Each of the six possible pairs of alleles was found to be present at random in the $F_{1}\left(\chi^{2}\right.$ for independent assortment of the pairs of alleles: $\chi_{5}^{2}=3.7, P=0.6$ ); this pattern of segregation best fits an autopolyploid type of inheritance. Disomic inheritance would result in pairs of alleles being in repulsion, and therefore mutually exclusive, in the $F_{1} s$.

\section{Discussion}

\section{One gene or several genes for diplosporous apomixis?}

Various models have been proposed to explain the regulation of apomixis. Most involve the existence of apomixis allele(s); that is, alleles that would be present in apomictic plants, and absent or silent in sexual plants. Under this assumption, apomixis would have arisen as the result of mutations at one or several loci playing a role in the reproductive process. The number of such loci is still strongly debated, but recent data, obtained in Panicum (Savidan, 1982), Ranunculus (Nogler, 1984a) or Brachiaria (do Valle \& Savidan, 1996), tend to demonstrate that apomeiosis is controlled by a single dominant gene. The control of parthenogenesis remains unclear, and it is still an open question whether the overall process relies on several distinct genetic controls, or can be traced back to a single mode of regulation. Several authors (Savidan, 1982; Mogie, 1992; Leblanc \& Savidan, 1994), have hypothesized that no specific genes are required for the parthenogenetic development of the unreduced gametes, the initiation of parthenogenesis being understood as a consequence of the shortening of megasporogenesis resulting from apomeiosis. Thus, parthenogenesis would be a pleiotropic consequence of apomeiosis, so that the whole developmental process of apomictic reproduction would rely on the control of apomeiosis, which is inherited as a Mendelian factor.

Normal recombination in the segregating populations, however, is a condition for the validity of the above genetic analyses. In our data, recombination in the chromosome carrying the 'apomixis gene(s)' is strongly restricted at the tetraploid (apomictic) level as opposed to the diploid (sexual) level in Tripsacum and maize, as shown by their RFLP maps. Furthermore, other RFLP loci have been located on that specific chromosome that are not linked to apomixis (data not shown), suggesting that recombination is restricted only locally. This particular behaviour cannot be directly generalized beyond the progenitor used in this study. Nevertheless, similar efforts to map apomixis gene(s) in other species suggest that a restricted level of recombination specifically on the segment controlling apomeiosis can be found in other situations, like Pennisetum (P. Ozias-Akins and D. Roche, pers. comm.).

Given this restricted level of recombination, the classical model of monogenic inheritance for apomixis probably needs more careful analysis:

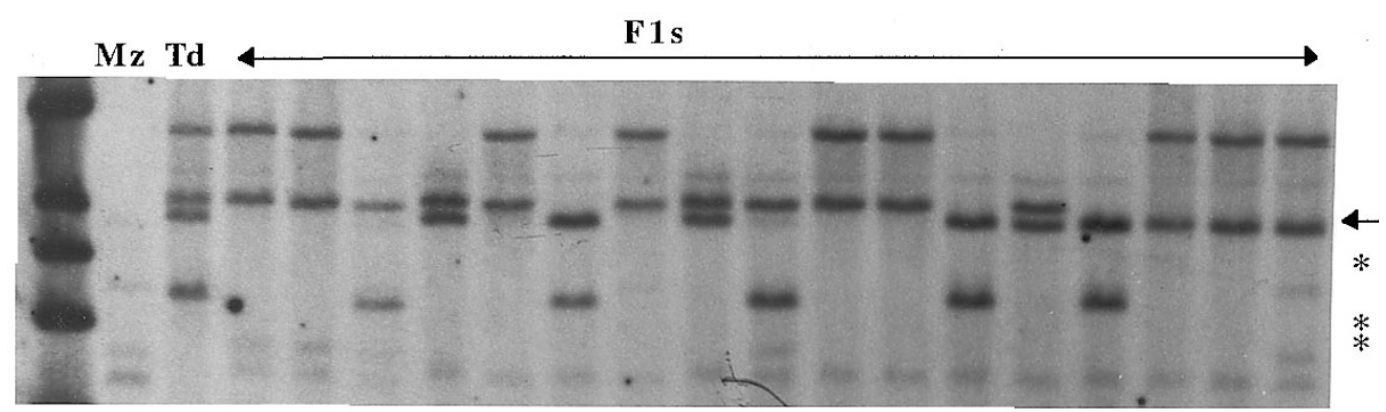

Fig. 2 Segregation of the four RFLP alleles revealed by umc 28 in the maize by tetraploid Tripsacum $\mathrm{F}_{1}$ population, digested by EcoR1. 'Td' is the Tripsacum parent; 'Mz' is the maize parent. The band cosegregating with diplospory is indicated by the arrow. ${ }^{*}$ indicates the segregating maize alleles. 
whatever the number of genes involved, they certainly behave as a single locus in small segregating populations. Our observations do not prove the existence of several genes, but at least suggest the possibility of a cluster of linked and co-adapted loci. Clearly, conventional segregation analyses in apomicts are not sufficient to conclude that a singlegene model is correct.

If it could be confirmed that apomixis depends on a more complex mode of regulation than previously thought, this would have strong implications on current efforts aiming at the isolation of the genetic system responsible for apomixis and its transfer to crops (Savidan \& Dujardin, 1992; Hanna, 1995; Calzada et al., 1996). Basically two options have been considered to generate new apomictic crops. The first one is to introduce the gene or genes responsible for apomixis into a sexual crop, either through wide hybridization between a sexual plant and an apomictic relative, or through genetic engineering using cloned apomixis genes. The second one is to generate apomixis de novo in a sexual plant through the appropriate mutation scheme. Both mutagenesis and gene-cloning strategies imply that a simple mode of regulation is responsible for apomixis, and would probably yield disappointing results in the case of a complex genetic system. The wide-hybridization approach depends less on the manner of regulation, the character being inherited as a block whatever the number of loci actually involved.

The bases of this restricted recombination remain unclear. Examples of blocks of cosegregating genes are extremely common in and outside the plant kingdom [several examples can be found in Dobzhansky (1970) or Grant (1975)]. The component genes might or might not be functionally related, but the group can form a favourable adaptive gene combination. The integrity of the block of genes is usually preserved through the establishment of factors that locally prevent recombination, among which chromosomal structural rearrangements, for example inversion or translocation heterozygotes, are common. In the case of tetraploid apomictic Tripsacum, though, no cytological data are available that would support the hypothesis of chromosomal rearrangements on the segment controlling apomixis.

\section{Allopolyploid vs. autopolyploid segregation}

A second category of hypotheses concerning the genetic control of apomixis postulates that no specific mutation(s) are required to induce apomixis.
The oldest version of these alternative hypotheses (Ernst, 1918) proposed that apomixis arose spontaneously as a result of disturbed sexual reproduction following interspecific hybridization. In a modern reformulation of the same concept, it has been suggested (Carman, 1997) that apomixis might be caused by the superposition of asynchronous signals in the female reproductive pathway, coded by the duplicated segments contributed by the two progenitors in an allopolyploid apomict. In the asynchrony hypothesis, allopolyploidy is therefore involved as a causal factor. That the vast majority of apomictic species are actually of allopolyploid origin strongly supports this model (Gustafsson, 1946-47; Asker \& Jerling, 1992). Other supporting evidence can be found in the work of Ellenström \& Zagorcheva (1977), who obtained apomictic allopolyploid genotypes of Raphanobrassica resulting from hybridization between duplicated sexual diploid accessions.

The results reported here demonstrate autopolyploidy specifically for the segment controlling apomixis in Tripsacum. This supports observations of chromosome pairing in maize-Tripsacum $\mathrm{F}_{1}$ hybrids, suggesting autopolyploidy on a whole-genome basis (de Wet et al., 1973). Furthermore, other examples of autopolyploid behaviour in apomicts have been reported, as in Panicum (Savidan, 1982). It is conceivable that those autopolyploids might represent inter-racial allopolyploidy, more than strict autopolyploidy. In both cases, though, those observations seem to contradict the idea that apomixis would result from hybridization of distant taxa.

\section{Comparative mapping between maize and Tripsacum}

Another approach to characterizing the gene(s) controlling apomixis can be suggested from the data presented here, using comparative mapping between maize and Tripsacum. It implies the identification in maize of the orthologous loci of the apomixis gene(s) expressed in Tripsacum. Two genes are said to be orthologous, as defined by Rieger et al. (1976), when they diverge from a common gene at the same time that the species harbouring them diverged. The great amount of comparative mapping performed during recent years (reviewed by Moore et al., 1995) has demonstrated a very high level of genome conservation among grasses. The mapping data reported here confirm a good level of conservation between maize and Tripsacum, at least in the chromosome segment controlling apomixis. Even though local rearrangments have certainly occurred, as suggested by our data, the same markers detect 
the same linkage groups in maize and Tripsacum, meaning that the segment controlling apomixis in Tripsacum probably exists in maize. Therefore, it is reasonable to suggest that the gene(s) involved in the expression of apomixis in Tripsacum might have one or several orthologous locations in the maize genome. Thus, by mapping the same set of probes in both Tripsacum and maize, it is theoretically possible to identify 'candidate genes' - that is, meiotic mutants already known in maize that would have: (i) the corresponding map position; and (ii) a phenotype suggesting orthology with the apomixis locus. A search in the maize mutant catalogue, through the Maize database (MaizeDB), yielded two distinct mutants, afd1 (absence of first division) and ell (elongate) that appear as interesting candidates. The afd1 mutation results in a diplosporous-like phenotype (Curtis \& Doyle, 1991; Golubovskaya et al., 1992) but without further initiation of embryo sac development, the megaspore mother cell being unable to act as an unreduced megaspore. The elongate (el1) mutant results in the production of a variable number of unreduced but fertilized gametes, and therefore triploid embryos (Rhoades \& Dempsey, 1966). Both mutant loci have been mapped to chromosome arms using B-A translocation stocks. The afd1 locus is located on maize chromosome 6L (B. Sheridan, pers. comm.), and el1 is on chromosome $8 \mathrm{~L}$ (Maize Genet. Coop. Newsletter 70, 1996, pers. comm.). Both $6 \mathrm{~L}$ and $8 \mathrm{~L}$ contain mapped loci linked with diplospory in Tripsacum. To determine whether these loci might be orthologous to the locus controlling diplospory, fine genetic mapping has been undertaken.

\section{Conclusions}

The results reported here support the onedominant-gene hypothesis for the failure of meiosis. At the same time, they also suggest that the overall apomictic process might be rather more complex, and potentially controlled by a cluster of linked genes. Finally, the data presented here prove the tetrasomic inheritance of the apomictic locus, thus questioning the allopolyploid origin of apomictic species.

Could the data presented here be generalized beyond Tripsacum? A remarkable diversity exists in the apomixis process, and even though they have the same consequences, apospory or diplospory involve quite different developmental processes. Nevertheless, the information reported here can be used to assess the genetic relatedness of the different forms of apomixis. Because many apomictic species belong to the grass family, comparative mapping studies could be undertaken to locate the gene(s) for diplospory and apospory in different genera. Such studies might uncover orthologous relationships between diplospory and apospory, and their numerous variations, thus revealing whether they correspond to a differential expression of the same genetic system, or to unrelated control mechanisms.

\section{References}

ASker, s. And Jerling, L. 1992. Apomixis in Plants. CRC Press, Boca Raton, FL.

CAlzAdA, J. P. V., CRANE, C. F. AND STElly, D. M. 1996. Apomixis: the asexual revolution. Science, 274, 1322-1323.

CARMAN, J. G.. 1997. Asynchronous expression of duplicated genes in angiosperms may cause apomixis, bispory, tetraspory and polyembryony. Biol. J. Linn. Soc., 61, 51-94.

CURTIS, C. A. AND DOYle, G. G. 1991. Double meiotic mutants of maize: implications for the genetic regulation of meiosis. J. Hered., 82, 156-163.

DOBZHANSKy, T. 1970. Genetics of the Evolutionary Process. Columbia University Press, New York.

ELlenström, s. AND ZAGORCHEVA, L. 1977. Sterility and apomictic embryo sac formation in Raphanobrassica. Hereditas, 87, 107-120.

ERNST, A. 1918. Bastardierung als Ursache der Apogamie im Pflanzenreich. Fischer, Jena.

GOLUBOVSKAYA, I., AVALKINA, N. A. AND SHERIDAN, W. F. 1992. Effects of several meiotic mutations on female meiosis in maize. Devl. Genet., 18, 411-424.

GRANT, v. 1975. Genetics of Flowering Plants. Columbia University Press, New York.

gustafsson, A. 1946-47. Apomixis in higher plants. Lunds Univ. Arsskr., 42, 1-67, 1946; 43, 71-179, 1947; 43, 183-370, 1947.

HANNA, w. W. 1995. Use of apomixis in cultivar development. Adv. Agron., 54, 333-354.

HOISINGTON, D., KHAIRALLAH, M. AND GONZÁLEZ-DE-LEÓN, D. 1994. Laboratory Protocols: CIMMYT Applied Molecular Genetics Laboratory, 2nd edn. CIMMYT, México, DF.

LEBLANC, O. AND SAVIDAN, Y. 1994. Timing of megasporogenesis in Tripsacum species (Poaceae) as related to the contol of apomixis and sexuality. Polish Bot. Stud., 8, $75-81$.

LEBLANC, O., GRIMANELLI, D., GONZALÉZ DE LÉON, D. AND SAVIDAN, Y. 1995a. Detection of the apomictic mode of reproduction in maize-Tripsacum hybrids using maize RFLP markers. Theor. Appl. Genet., 90, 1198-1203.

LEBLANC, O., PEEL, M. D., CARMAN, J. G. AND SAVIDAN, Y. 1995b. Megasporogenesis and megagametogenesis in several Tripsacum species (Poaceae). Am. J. Bot., 82, $57-63$.

MOGIE, M. 1992. The Evolution of Asexual Reproduction in Plants. Chapman \& Hall, London.

(C) The Genetical Society of Great Britain, Heredity, 80, 33-39. 
MOORE, G., DEVOS, K. M., WANG, Z. AND GALE, M. D. 1995. Grasses: line-up and form a circle. Curr. Biol., 5, 738-742.

NOGLER, G. A. 1984a. Genetics of apospory in apomictic Ranunculus auricomus V. Conclusions. Bot. Helvet., 94, 411-422.

NOGLER, G. A. 1984b. Gametophytic apomixis. In: Johri, B. M. (ed.) Embryology of Angiosperms, pp. 475-518. Springer-Verlag, Berlin.

RENNER, O. 1916. Zur Terminologie des pflanzlichen Generationswechsels. Biol. Zbl., 36, 337-374.

RHOADES, M. M. AND DEMPSEY, E. 1966. Induction of chromosome doubling at meiosis by the elongate gene in maize. Genetics, 54, 505-522.

RIEGER, R., MICHAELIS, A. AND GREEN, M. M. 1976. Glossary of Genetics and Cytogenetics. Springer, Berlin.

RITTER, E., GEBHARDT, C. AND SALAMINI, F. 1990. Estimation of recombination frequencies and construction of
RFLP linkage maps in plants from crosses between heterozygous parents. Genetics, 125, 645-654.

SAVIDAN, Y. 1982. Nature et hérédité de l'apomixie chez Panicum maximum Jacq. Ph.D. Dissertation, University of Paris XI.

SAVIDAN, Y. AND DUJARDIN, M. 1992. Apomixie. La prochaine révolution verte? La Recherche, 241, 326-334.

StebiIns, G. L. 1950. Variation and Evolution in Plants. Columbia University Press, New York.

DO VALle, C. B. AND SAVIDAN, Y. H. 1996. Genetics, cytogenetics and reproductive biology of Brachiaria. In: Miles, J. W., Maass, B. L. and do Valle, C. B. (eds) Brachiaria: Biology, Agronomy, and Improvement, pp. 147-163. CIAT-EMBRAPA, Cali, Colombia.

DE WET, J. M. J., HARLAN, J. R., ENGLE, L. M. AND GRANT, C. A. 1973. Breeding behavior of maize-Tripsacum hybrids. Crop Sci., 13, 254-256. 